\title{
Developing a psychotropic medication education group on a secure ward for men with intellectual disabilities
}

Rory Sheehan, John Rochester, Fatima Hafesji, Rita Kyambadde, Shaun Gravestock

\begin{abstract}
Purpose - To establish and evaluate a psychotropic medication education group for men with intellectual disability on a secure psychiatric ward.
\end{abstract}

Design / method / approach - A multidisciplinary team was convened to oversee the project. A curriculum was developed that covered major classes of psychotropic drugs as well as broader themes related to taking medication and general wellbeing. Each group session incorporated a range of teaching methods supported by accessible materials. Evaluation was by qualitative and quantitative methods.

Findings - There was interest and enthusiasm for the group. Participant feedback was generally positive and most of those who completed the group reported achieving their personal learning goals. There was no significant difference in results of a medication knowledge test at baseline and at the end of the course. Feedback from group members and reflections of the course facilitators are discussed.

Limitations - The results of this small-scale study may not be applicable to other groups or settings. Evaluation measures seemed unable to capture some elements of the group processes and outcomes.

Practical implications - Establishing and running a psychoeducational group on a low-secure ward for men with intellectual disabilities is possible and potentially valuable. Learning from this project will be useful for others considering group-based interventions for people with intellectual disability. 
Originality - This paper adds to the limited literature describing interventions to improve medication knowledge in people with intellectual disability.

\section{Keywords}

Intellectual disability, psychotropic medication, education, group intervention, service evaluation, feasibility study

\section{Article classification}

Case study 


\section{Developing a psychotropic medication education group on a secure ward for men with intellectual disabilities}

\section{Background}

There is ongoing controversy over the use of psychotropic medications for people with intellectual disability. Recent epidemiological studies utilising routinely-collected data from large primary care databases have highlighted notable discrepancies between the number of people with intellectual disability who are prescribed psychotropic medication and the number with a recorded mental illness for which it is licensed (Sheehan et al., 2015, Public Health England, 2015). In many cases, it seems that psychotropic medication is used to manage challenging behaviour; this is not necessarily inappropriate, but the evidence to support psychotropic drug use for challenging behaviour is lacking and many fear that a reliance on psychotropic medication detracts from the delivery of psychosocial interventions that might more effectively target the underlying cause of the behaviour and are necessary to improve quality of life.

In England there is now a national initiative to reduce psychotropic medication prescribing for people with intellectual disability. The majority of attention has focused on antipsychotic medications, as the class of psychotropic that has historically been associated with greatest degree of prescribing and arguably has the most significant side-effect profile. However, the reduction or withdrawal of antipsychotic medication used for challenging behaviour risks harms as well as having potential benefits. A recent systematic review concluded that decisions to reduce antipsychotic medication used for challenging behaviour should be made on an individual basis and highlighted that setting and support factors must be optimised if attempts to withdraw antipsychotics used for challenging behaviour are to be successful (Sheehan and Hassiotis, 2016). Indeed, the NICE guidelines on management of challenging behaviour and prescribing guidance issued by the Royal College of Psychiatrists both contain a place for antipsychotic prescribing in challenging behaviour and the absence of mental illness, if supplemented by frequent medication review and the provision of additional multidisciplinary interventions (National Institute for Health and Care Excellence 2015, Royal College of Psychiatrists, 2016). 
This renewed focus on psychotropic medication use in people with intellectual disability has prompted reflection within clinical teams and motivated attempts to ensure that psychotropic medication is used in the most appropriate way. In this paper we describe a practice initiative aimed at improving knowledge and understanding of psychotropic medication in adult men with intellectual disability who are detained in a low secure psychiatric setting.

\section{Introduction}

People with intellectual disability should be informed about their treatment but often have poor knowledge and understanding of prescribed medication (Hall and Deb, 2008, Arscott et al., 2000, Crossley and Withers, 2009, Heslop et al., 2005). If patients are to offer valid consent, they must have at least a basic understanding of the treatment (including likely benefits and potential harms) and its alternatives (General Medical Council, 2006). Even in those who lack capacity to consent, it is considered good practice to provide information up to their level of understanding and to facilitate engagement in treatment decisions as far as possible. Despite this, and the central role that psychotropic medication often has in management of people with intellectual disability and mental illness, few methods aimed at improving the knowledge of people with intellectual disability have about prescribed medication have been described and formally evaluated (Strydom and Hall, 2001, Aman et al., 2007, Ferguson and Murphy, 2014). The consequences of poor health literacy are likely to include reduced satisfaction and treatment compliance, as well as medication errors and safety incidents (such as receiving too much, or too little medication).

Psychoeducational groups have been developed in inpatient intellectual disability services and have a limited, but growing, evidence base to support their effectiveness (Rose et al., 2014, Crowley et al., 2008, Douds et al., 2014). There are several advantages to group interventions, leading some authors to suggest that they are an under-utilised treatment modality (Fletcher, 1993). Group interventions can act as a social support system and discussion within the group allows for vicarious learning. They can be an efficient use of 
resources and staff time as they allow several people to receive an intervention concomitantly. Some people with intellectual disability might prefer learning in group settings, where they can involve themselves to a degree that feels comfortable, over individual sessions where they might feel more pressure to perform. However there are also drawbacks to group interventions including loss of privacy and potential exposure of personal experiences and feelings. People with social impairments or anxiety may find accessing groups difficult and it is possible that personality clashes or tensions between group members might hinder the progress of the group (Gravestock and McGauley, 1994, British Psychological Society, 2016).

Psychotropic medication education groups are a relatively common feature of the therapy programme offered in secondary care services and have been reported in a number of settings serving different patient groups (Gavin and Frey, 2012, Farkas, 1990, Kuipers et al., 1994, Kavanagh et al., 2003, Bastiaens, 1992, Eisenmann, 2012). Several studies that evaluated the group intervention have reported modest benefits in knowledge (Farkas, 1990, Kavanagh et al., 2003), self-reported attitudes (Neizo and Murphy, 1983), and psychotropic medication compliance (Robinson et al., 1986), although other studies have failed to demonstrate benefit (Kuipers et al., 1994, Gavin and Frey, 2012). Given the diversity in delivery of the interventions and of the groups targeted, the variation in evaluation measures, and the necessary differences in educating people with intellectual disability, it is difficult to generalise results from existing literature and extrapolate them to our circumstances.

To our knowledge there are no published reports of a medication education group in a specialist in-patient unit for adults with intellectual disability. The work that is probably most relevant to the current project is that of Ferguson and Murphy, who assessed the effect of training on the ability of adults with intellectual disability living in the community to give consent to their medication; after three group training sessions participants demonstrated greater ability to give consent to their prescribed medication (Ferguson and Murphy, 2014).

We describe a psychoeducational group intervention on a ward which provides care for 15 adult men with mild-moderate intellectual disability and a range of complex mental health problems associated with offending or challenging behaviour. Many patients on the ward 
have multiple diagnoses including mental illness, personality disorder, and autism spectrum conditions. The ward offers assessment and intensive treatment from a multi-disciplinary staff team with a focus on recovery and risk-reduction. All patients are detained under civil or forensic Sections of the Mental Health Act (1983) and the majority are prescribed psychotropic medication.

Our aim was to empower patients as partners in their care by increasing knowledge and understanding about psychotropic medication. We determined to achieve this by establishing a ward-based psychotropic medication education group that would provide a safe and supportive environment for patients to learn, discuss, and exchange information related to medication and mental wellbeing and recovery more generally.

\section{Method}

Course development

A multi-disciplinary project team consisting of clinicians with experience working with people with intellectual disability was convened to oversee the project. A curriculum was devised that would cover all important psychotropic medication groups, as well as broader themes around medication use such as capacity and consent (table 1). For each class of medication we discussed indications, how they could help, possible side-effects along with how to manage these, monitoring requirements, and where to seek further information (Howland, 2009). The group ran weekly for eight sessions.

[Figure 1 near here]

Figure 1 Course curriculum

Most sessions were configured as semi-structured discussions led by the course primary facilitators (SG and RS). We endeavoured to create an informal atmosphere that allowed flexibility to respond to participant needs whilst maintaining overall group direction and ensuring fundamental material was covered. We were mindful of the difficulties that 
participants might have with didactic and 'classroom style' teaching and incorporated a range of learning methods to maintain interest (Primeau and Frith, 2014). Sessions were interactive and participants were encouraged to share their own experiences, where they felt comfortable to do so.

Additional materials were provided to supplement the discussion and as a resource that participants could refer to between sessions. These included the group contract and rules, personal copies of individual's own drug charts, accessible information leaflets, and course worksheets, which participants kept in individual folders. Where suitable freely-available resources were available these were used and in other cases new materials were developed.

Homework was assigned after three sessions. Tasks included making a list of the psychotropic medication prescribed and obtaining a leaflet for one of the medications. In week 6 participants were asked to choose a topic of particular interest that could be delivered as a five-minute presentation to the rest of the group (figure 2).

Positive reinforcement was used to motivate participants. A small prize was given for the best presentation and certificates were awarded at the end of the course.

[Figure 2 near here]

Figure $\mathbf{2}$ Topics chosen for microteachings

Recruitment

The group was advertised at the ward community meeting for several weeks prior to starting. Prospective participants were approached individually and the purpose of the group was explained in more detail. Attendance was voluntary and a contract was signed between the course lead and the participant. 
We allowed patients who had not signed up to the group to attend a one or two sessions with the intention that these would act as an introduction and facilitate full participation in the next round of the group.

\section{Evaluation measures}

A range of outcomes were of interest in this study and evaluated using different means.

Participants were asked for their positive and negative experiences of the group in verbal feedback sessions half way through (week 4) and at the end of the course (week 8).

Facilitators spent time in a 'debrief' following each group session and reflected on what had worked well and any difficulties encountered. Records were kept and discussed with the project team.

The effectiveness of the group in improving participant knowledge and confidence with prescribed medication was assessed by self-report and also by a test of medication knowledge. Participants were asked to rate their agreement with the following questions on a 5 point Likert scale (from "strongly disagree" to "strongly agree"):

- I know enough about my medication (self-rated knowledge)

- I am able to ask staff about my medication (self-rated skills)

- I think medication is an important part of my treatment (attitude)

Group members were supported to develop 2 or 3 personal learning objectives. The degree to which participants felt they had achieved these by the end of the course was tested using the same Likert scoring system described above.

A test of medication knowledge was developed consisting of 20 statements that participants were asked to rate as 'true' or 'false'. True and false statements were randomly distributed and the content of the quiz was covered in the course curriculum. Participants completed the same quiz at the beginning and end of the course. 


\section{Results}

Recruitment and participants

Six patients were recruited to the group (mean age 31 years, standard deviation 13.3). Participants had a range of psychiatric diagnoses and were at various stages in their recovery journey, although none were acutely mentally unwell at the time that the group was run. All were prescribed regular psychotropic medication for mental illness. Most participants attended all eight group sessions (median 8; range 6-8). The group ran on the ward on a Thursday morning between November 2016 and January 2017.

\section{Qualitative feedback}

Overall responses from participants about the acceptability of the group were positive and most of those who attended indicated that they found the group interesting and useful. A number of participants made suggestions for improvements to the group (figure 3 ).

[Figure 3 near here]

Figure 3 Participant feedback on the psychotropic medication education group

\section{Quantitative results}

Participants rated their knowledge of and comfort with medication highly at baseline; all participants reported they "strongly agree" with the statements "I know enough about my medication" and "I am able to ask staff about my medication," and five out of the six strongly agreed with the statement "Medication is an important part of my treatment". There were few differences by the end of the group in the self-report measures. 
Five out of the six participants "strongly agreed" that they had met their personal objectives by attending the group, with the remaining participant indicating that he was "not sure" if they had been met.

The psychotropic medication knowledge test was completed by all participants. Mean baseline score was 13.7 (standard deviation 1.862) and mean score at the end of the group was 13.3 (standard deviation 2.066). A paired Student's t-test confirmed no statistically significant difference between pre- and post-group scores ( $\mathrm{t}=0.250 ; p=0.813)$ (figure 4).

[Figure 4 near here]

Figure 4 Scatter plot showing pre- and post-group scores on the medication knowledge quiz $(n=6)$.

\section{Discussion}

Methods for evaluating psychoeducational groups are not well described (Brown, 2005). In this paper we focused on several aspects of feasibility, that is; practical considerations of how to design and implement the intervention; the testing of measurement tools; and estimating parameters related to demand, acceptability and potential outcomes (Bowen et al., 2009).

Demand We were pleasantly surprised to have experienced no difficulty in recruiting several ward patients to the group. Participants showed willingness to learn more about their medication and asked pertinent questions. There were a small number of patients on the ward who were too mentally unstable to participate and who were not recruited to the group.

Acceptability We found that the medication education group was acceptable to participants.

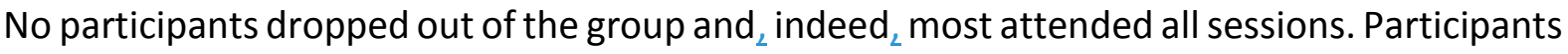
reported both during formal feedback and informally that they had enjoyed the group. There were some useful suggestions for improvement which will be considered in future iterations of the group. One participant asked for sessions to be shortened to 30 minutes, and another requested refreshments be provided. Some participants felt annoyed with the small number 
of people who were not members of the group but who attended one or two sessions to get a 'taster' of the group; these people tended to be less well and were perceived as disruptive.

Practicality We anticipated some challenges in the practical aspects of running a new group on the ward. The group ran at 10am so as not to conflict with planned patient leave and other activities but a number of participants found it difficult to get ready for this time and the group often started a few minutes late.

Running the group required no specific funding but did draw on the time and resources of several members of the team.

Integration A key question in this evaluation was how the medication group would fit with the demands and constraints already placed on the ward staff, patients, and the activity and therapy timetable. We identified a morning session during which a suitable room was available and when there were few other scheduled activities, however there were inevitably some clashes and a small number potential participants that were not able to attend.

Limited efficacy testing We did not demonstrate improvements in medication knowledge or beliefs about medication in the qualitative evaluation. This contrasts with the observations of the facilitators and the qualitative feedback received that suggested the group had positively impacted medication knowledge, and may be due to unsuitable instruments being used to measure outcomes (see limitations, below).

Attending the group is also likely to have contributed to benefits in other, more nebulous domains that are difficult to quantify, such as improved therapeutic relationships and patient satisfaction.

We know from evaluation of medication education groups in other populations that they can be an effective way of imparting knowledge, and can lead to tangible improvements in patient outcomes (Farkas, 1990, Kavanagh et al., 2003, Neizo and Murphy, 1983, Robinson et al., 1986). However, as is common in many complex interventions, extracting the elements that 
are essential for success is not straightforward and the current literature in this field is not sufficient to inform the design of the group.

Running an educational group for people with intellectual disability adds an additional layer of complexity to the intervention, as limitations in cognitive ability can impact learning and differing strengths and needs amongst group members (for example, in literacy) is likely to be significant. Further experience of running the group and the introduction of creative and novel techniques will hopefully reveal the most effective methods of teaching and learning in this group. Giving people information only on the medications they are taking might be a more realistic way of improving necessary knowledge while avoiding information overload (Farkas, 1990).

It is also possible that we did not observe an improvement in medication knowledge following the group as the cohort who attended were already interested in medication and had reasonably good baseline knowledge. Although the quiz results do not appear to support this, it is possible that our intervention was aimed at a level that was too elementary.

\section{Facilitator reflections}

A number of key themes emerged through discussions and debrief sessions with the project team.

It was found to be vital to involve the wider multi-disciplinary team if a new group is to become fully embedded in an environment where there are competing demands for resources and attention. Ward staff helped in developing the group and supporting participants, both within and outside the group and we noticed that the group ran more smoothly when those with additional needs were supported by extra staff members joining the group. Group work might raise several issues that participants wish to discuss with staff outside group sessions, and staff can help to reiterate important messages at other times, for example, when administering medication or opportunistically. We found that participants did 
not often do the homework that was set and we suggest in future groups that this supported by the ward team.

The time commitment involved in developing materials, recruiting participants, running and evaluating the group was significant and probably greater than initially expected. Even seemingly simple practical issues require organisation and sometimes negotiation; advance planning and contingency arrangements are necessary.

The primary facilitators (RS and SG) were both psychiatrists who worked on the ward and the potential for this to influence group dynamics and motivation to join the group was considered. Our impression was that participants were open and honest in discussing their medication and experiences, including voicing negative attitudes where held, and the presence of the medical team did not inhibit frank discussion or encourage acquiescence. Nonetheless, we must be mindful that the group was not an entirely neutral space and the other roles of the facilitators could present a distraction.

We learned that facilitating a group requires particular skills that might not routinely be taught to healthcare professionals. Running the group was challenging but rewarding; sessions were enjoyable and resulted in an understanding of medication issues and patient perspectives that might not otherwise have been obtained.

Enforcing boundaries and rules was sometimes difficult, particularly in the initial sessions when roles and responsibilities were being established. Over time a shared understanding did develop but the group always relied on a relatively high level of guidance and direction from the facilitators.

Participants were known to each other prior to attending the group. This could have been a facilitator or a hindrance to the work of the group but our experience was that any preexisting interpersonal difficulties did not have a significant impact in the group sessions and few overt tensions between members were witnessed. 
Participants attending this group did so voluntarily and tended to be relatively highly motivated. Other strategies (such as individual psychoeducation) are likely to be needed to deliver medication education to those patients who are less engaged or unable to participate in group learning.

In this feasibility study it was expected that the group would develop naturally over time in response to feedback and facilitator reflections. We found it helpful to be open to feedback and flexible in how the group was delivered. Using a range of materials was helpful but we did also wonder whether additional methods for participant engagement could be employed, such as role plays/simulation, audio-visual materials, and creating artwork.

\section{Limitations}

This study reports the results of a small-scale intervention on a single-sex specialist ward and the findings might not be applicable to other settings or groups. However, we believe there are certain learning points which will be relevant to those establishing other psychoeducational groups for people with intellectual disability in in-patient and possibly also community settings (figure 5).

Although efficacy testing was not a primary aim of this work, we failed to demonstrate any improvement in knowledge or beliefs about medication in the participants who attended the group. The group was too small to form any subgroups (either by IQ level or by diagnosis) that might be used to determine people for whom it is most effective.

Our outcome measures appear not to have been sensitive enough to capture the learning and group processes that the qualitative feedback suggested had occurred. Participants seemed to over-estimate their knowledge and skills at baseline which made demonstrating improvement impossible. It is possible that the Likert scale questions encouraged acquiescence in the way that they were phrased. Similarly, the 'true/false' test of medication knowledge, although written with care, might not have been the best method to use in a group of intellectually disabled persons who may be quite suggestible. 
When planning the group we looked for a validated and objective measure of medication knowledge for people with intellectual disability but found that none existed; we therefore created our own quiz, run in a 'true or false' format, that covered the content of the group. As other authors have highlighted, this style of quiz is likely to result in a $50 \%$ score even if respondents guess the answer to every question, leading to inflated pre-group assessment and limiting the scope for improvement over time (Burns et al., 2011).

To build on this work, further investigation focusing on outcomes is necessary, possibly by including proxy measures of patient medication knowledge. It is also important to establish whether improvements in medication knowledge translate to changes in behaviour or other important outcomes such as adherence and satisfaction, and if knowledge gained in a group is retained in the longer-term (British Psychological Society, 2016).

[Figure 5 near here]

Figure $\mathbf{5}$ - Learning points for running education groups for people with intellectual disability

\section{Conclusion}

People with intellectual disability should have information about their treatment and the medication they take. Our work shows that it is possible to run a psychoeducational group for people with intellectual disability and complex mental health needs in a low secure inpatient setting. Recognising the limitations of this feasibility study, the results of qualitative evaluation are encouraging; it seems that the group was valued and has the potential to deliver benefits. Further work is needed to establish outcome measures that can fully capture the group processes and outcomes in different domains. A number of practical considerations have been identified that will inform future iterations of the medication group and can be used by others to guide the development of psychoeducational groups in this population. 



\section{References}

Aman, M. G., Benson, B. A., Farmer, C. A., Hall, K. L., Malone, K. M. \& Taylor, S. J. 2007. Project MED: Effects of a Medication EDucation Booklet Series for Individuals With Intellectual Disabilities. Intellectual and Developmental Disabilities, 45, 33-45.

Arscott, K., Stenfert Kroese, B. \& Dagnan, D. 2000. A study of the knowledge that people with intellectual disabilities have of their prescribed medication. Journal of Applied Research in Intellectual Disabilities, 13, 90-99.

Bastiaens, L. 1992. The impact of an intensive educational program on knowledge, attitudes, and side effects of psychotropic medications among adolescent inpatients. Journal of Child and Adolescent Psychopharmacology, 2, 249-258.

Bowen, D. J., Kreuter, M., Spring, B., Cofta-Woerpel, L., Linnan, L., Weiner, D., Bakken, S., Kaplan, C. P., Squiers, L. \& Fabrizio, C. 2009. How we design feasibility studies. American Journal of Preventive Medicine, 36, 452-457.

British Psychological Society. 2016. Psychological Therapies and People who have Intellectual Disabilities. Leicester: The British Psychological Society.

Brown, N. 2005. Psychoeducational groups. In: Wheelan, S. (ed.) The Handbook of Group Research and Practice. Sage Publications.

Burns, J., Aspinall, C. \& Matthews, C. 2011. An evaluation of an alcohol awareness group for learning disabled offenders in a secure setting. Journal of Learning Disabilities and Offending Behaviour, 2, 159-166.

Crossley, R. \& Withers, P. 2009. Antipsychotic medication and people with intellectual disabilities: their knowledge and experiences. Journal of Applied Research in Intellectual Disabilities, 22, 77-86.

Crowley, V., Rose, J., Smith, J., Hobster, K. \& Ansell, E. 2008. Psycho-educational groups for people with a dual diagnosis of psychosis and mild intellectual disability: a preliminary study. Journal of Intellectual Disabilities, 12, 25-39.

Douds, F., Mckechanie, A., Simpson, Y. \& Murphy, L. 2014. "Staying Well”: a psychoeducational group for people with an intellectual disability, co-morbid mental illness and offending behaviour. Journal of Intellectual Disabilities and Offending Behaviour, 5, 54-59.

Eisenmann, C. M. 2012. Revising a medication education program on an inpatient child and adolescent psychiatric unit. Journal of Psychosocial Nursing and Mental Health Services, 50, 41-47. 
Farkas, M. 1990. Utilizing the nursing process in the development of a medication group on an inpatient psychiatric unit. Perspectives in Psychiatric Care, 26, 12-17.

Ferguson, L. \& Murphy, G. H. 2014. The effects of training on the ability of adults with an intellectual disability to give informed consent to medication. Journal of Intellectual Disability Research, $58,864-873$.

Fletcher R.J. 1993. Group therapy for persons with mental retardation. In: Fletcher R, Dosen A. (eds.) Mental Health Aspects of Mental Retardation. New York: Lexington Books.

Gavin, L. \& Frey, T. 2012. Assessment of a pharmacist-run medication education group for inpatient psychiatric patients. Mental Health Clinician, 2, 94-99.

General Medical Council 2006. Good Medical Practice. London: General Medical Council.

Gravestock, S \& McGauley, G. 1994. Connecting confusions with painful realities: Group analytic psychotherapy for adults with learning disabilities. Psychoanalytic Psychotherapy, 8, 153167.

Hall, S. \& Deb, S. 2008. A qualitative study on the knowledge and views that people with learning disabilities and their carers have of psychotropic medication prescribed for behaviour problems. Advances in Mental Health and Learning Disabilities, 2, 29-37.

Heslop, P., Folkes, L. \& Rodgers, J. 2005. The knowledge people with learning disabilities and their carers have about psychotropic medication. Tizard Learning Disability Review, 10, 10-18.

Howland, R. H. 2009. What should patients be told about their medications? Journal of Psychosocial Nursing and Mental Health Services, 47, 17-20.

Kavanagh, K., Duncan-Macconnell, D., Greenwood, K., Trivedi, P. \& Wykes, T. 2003. Educating acute inpatients about their medication: is it worth it? An exploratory study of group education for patients on a psychiatric intensive care unit. Journal of Mental Health, 12, 71-80.

Kuipers, J., Bell, C., Davidhizar, R., Cosgray, R. \& Fawley, R. 1994. Knowledge and attitudes of chronic mentally ill patients before and after medication education. Journal of Advanced Nursing, 20, 450-456.

National Institute for Health and Care Excellence 2015. Challenging behaviour and learning disabilities: prevention and interventions for people with learning disabilities whose behaviour challenges (NICE Guideline NG11). London: NICE.

Neizo, B. \& Murphy, M. K. 1983. Medication groups on an acute psychiatric unit. Perspectives in Psychiatric Care, 21, 70-73.

Primeau, M. S. \& Frith, K. H. 2014. Teaching Patients With an Intellectual Disability. Home Healthcare Now, 32, 249-250. 
Public Health England 2015. Prescribing of psychotropic drugs to people with learning disabilities and/or autism by general practitioners in England. London: Public Health England.

Robinson, G.L., Gilbertson, A.D. \& Litwack L. 1986. The effects of a psychiatric patient education to medication program on post-discharge compliance. Psychiatric Quarterly, 58, 113-118.

Rose, L., Breen, O. \& Webb, Z. 2014. An inpatient Healthy Living Group. Advances in Mental Health and Intellectual Disabilities, 8, 128-137.

Royal College of Psychiatrists 2016. Psychotropic drug prescribing for people with intellectual disability, mental health problems and/or behaviours that challenge: practice guidelines. London: Royal College of Psychiatrists.

Sheehan, R., Hassiotis, A., Walters, K., Osborn, D., Strydom, A. \& Horsfall, L. 2015 Mental illness, challenging behaviour, and psychotropic drug prescribing in people with intellectual disability: UK population based cohort study. British Medical Journal 351, h4326.

Sheehan, R. \& Hassiotis, A. 2016. Reduction or discontinuation of antipsychotics for challenging behaviour in adults with intellectual disability: a systematic review. Lancet Psychiatry, 4, 238-256.

Strydom, A. \& Hall, I. 2001. Randomized trial of psychotropic medication information leaflets for people with intellectual disability. Journal of Intellectual Disability Research, 45, 146-151. 